\title{
Attentional blink and selection in the tactile domain
}

\author{
R. Dell'Acqua \\ Department of Developmental Psychology, University of Padova, \\ Padova, Italy \\ P. Jolicœur \\ Department of Psychology, Université de Montréal, Montréal, Canada \\ P. Sessa \\ Department of Developmental Psychology, University of Padova, \\ Padova, Italy \\ M. Turatto \\ Department of Cognitive Science, University of Trento, Trento, Italy
}

\begin{abstract}
Brief tactile presses stimulated the index and middle fingers of the right and left hands. The stimulation on each hand consisted of a triplet of presses. Each triplet was composed of a brief press to one finger (e.g., the middle finger), followed by a brief press to the other finger (e.g., the index finger), and by a final simultaneous press to both fingers of a given hand. With equal probability, a triplet could begin with the index or middle finger, and either $360 \mathrm{~ms}$ or $800 \mathrm{~ms}$ later another triplet stimulated fingers on the other hand. The task was to indicate which finger was stimulated first in each triplet. In four experiments, response accuracy to the second triplet revealed an attentional blink in taction, that is, responses were less accurate at the short triplet-triplet interval than at the long triplet-triplet interval. This effect was substantially reduced when the first triplet could be ignored.
\end{abstract}

Even with little or no training, most observers have the remarkable ability to identify a visual target when it is embedded in a rapid serial visual presentation (RSVP) stream of spatially overlapping distractors displayed at rates of 10 items/s (e.g., Potter, 1976). Under optimal conditions, some

Correspondence should be addressed to Roberto Dell'Acqua, Dept. of Developmental Psychology, University of Padova, Via Venezia 8, 35131 Padova, Italy. E-mail: dar@unipd.it

This research was supported by a FIRB grant from the Italian Government awarded to RDA and a grant from the Natural Sciences and Engineering Research Council of Canada awarded to PJ. We thank Stefano Massaccesi, engineer at Department of General Psychology at University of Padova, for designing the tactile stimulators and their interface to the stimulation computer. 
observers can even identify well-learned targets presented at up to 100 items/s (Sperling, Budiansky, Spivak, \& Johnston, 1971). When identification is required for two targets, which we will designate as T1 and T2, respectively, identification of $\mathrm{T} 2$ is however often impaired relative to $\mathrm{T} 1$ or to a control condition in which only $\mathrm{T} 2$ is to be reported. This phenomenon occurs even when the rate of presentation is as slow as $7-10$ items/s (e.g., Arnell \& Jolicœur, 1999), and is typically observed when the stimulus-onset asynchrony (SOA) between T1 and T2 is shorter than $500 \mathrm{~ms}$. Furthermore, the duration of the effect appears to depend on the time spent processing T1 (e.g., Jolicœur, 1999a; Jolicœur, Dell'Acqua, \& Crebolder, 2000).

Several studies suggest that the cause of the AB resides at a postperceptual stage of processing of T2, namely, beyond the state at which T2 and the items composing the RSVP stream have been fully identified. Support for this view has come from AB paradigms using the RSVP of words. Using these paradigms, $\mathrm{T} 2$ report accuracy has been shown to be a function of the semantic relationship between $\mathrm{T} 1$ and $\mathrm{T} 2$, with higher $\mathrm{T} 2$ identification accuracy under conditions in which $\mathrm{T} 1$, or a distractor following $\mathrm{T} 1$ and preceding T2, was semantically related to T2 (Isaak, Shapiro, \& Martin, 1999; Juola, Duvuru, \& Peterson, 2000; Maki, Frigen, \& Paulson, 1997; Shapiro, Driver, Ward, \& Sorensen, 1997). Consistent with the behavioural evidence, full identification of a missed $\mathrm{T} 2$ during the $\mathrm{AB}$ has also been supported by studies using electrophysiological methods. Vogel, Luck, and Shapiro (1998, Exp. 2, p. 1662) have shown that, during the AB, N400 responses to missed $\mathrm{T} 2$ words were preserved in the event-related potential time-locked to T2 onset (see also Rolke, Heil, Streb, \& Hennighausen, 2001). A preserved $\mathrm{N} 400$ response during the $\mathrm{AB}$ suggests that $\mathrm{T} 2$ was processed to the level of meaning during the AB (e.g., Kutas \& Hillyard, 1980). In a different experiment (Exp. 4, p. 1666), Vogel et al. have found a reduced P300 response to an infrequent $\mathrm{T} 2$ letter stimulus presented during the $\mathrm{AB}$ (Exp. 4 , p. 1666). Although different interpretations have been proposed for the P300 response (e.g., Donchin, 1981; Verleger, 1988), there is a general consensus that the P300 represents electrophysiological evidence for the updating of information in short-term memory (STM; Donchin \& Coles, 1988; Johnson, 1986). Jointly with the preservation of N400 responses, the evidence of suppressed $\mathrm{P} 300$ responses during the $\mathrm{AB}$ have thus been taken to reflect a failure in the transfer of a postperceptual T2 to STM, a memory system hypothesised to support the overt report of T2 a few seconds later from its presentation (Chun \& Potter, 1995; Dell'Acqua, Jolicœur, Pesciarelli, Job, \& Palomba, 2003; Vogel et al., 1998). In vision, transfer, or consolidation, into STM appears to require a serial operation that constitutes a bottleneck in the processing of $\mathrm{T} 2$. When consolidation mechanisms are busy with $\mathrm{T} 1$ processing, the consolidation of a T2 presented shortly after $\mathrm{T} 1$ is hypothesised to be momentarily postponed. 
This delay in the consolidation of T2 explains the loss of T2 at short T1-T2 lags. Studies in which the masking parameters of $\mathrm{T} 1$ and $\mathrm{T} 2$ have been systematically manipulated have indeed suggested that the representation of T2 that has not already reached the status of STM trace is likely more susceptible to degradation (or substitution; Dell'Acqua, Pascali, Jolicœur, \& Sessa, 2003; Giesbrecht \& Di Lollo, 1998) by trailing items, compared to those in STM.

Recently, an interesting question concerning the $\mathrm{AB}$ phenomenon has focused on whether the $\mathrm{AB}$ is specific to vision, as opposed to the manifestation of a supramodal capacity limitation in the information processing system. In the supramodal view, the processing of stimulation from different sensory modalities would require access to one or more common amodal central mechanisms. These common central mechanisms would mediate the integration of multiple sensory inputs into a centrally unified stream of behaviour.

One way to study this issue, in the context of the $\mathrm{AB}$, has been to explore the $A B$ effect in sensory modalities other than vision. Several studies have investigated the $\mathrm{AB}$ effect in audition, with mixed results. Using the rapid auditory presentation (RAP) of stimuli, some researchers have found auditory AB effects (Arnell \& Jolicœur, 1999; Mondor, 1998), whereas others have not (Potter, Chun, Banks, \& Muckenhoupt, 1998). Chun and Potter (2001) attempted to sort out this inconsistency and suggested that nonvisual $\mathrm{AB}$ effects tend to be found only when there is a task switch (Allport, Styles, \& Hsieh, 1994; Rogers \& Monsell, 1995) associated with the processing of the two targets. That is, Chun and Potter (2001; see also Potter et al., 1998) proposed that pure (i.e., not affected by switch effects) AB effects are typical of the visual modality, whereas other modalities would in principle be insensitive to $\mathrm{AB}$ effects if there were complete homogeneity in the features allowing the selection of the two targets, and in the features reported from the two targets at the end of each trial. More recent work, however, has established important exceptions to Chun and Potter's (2001) proposal, insofar as pure auditory $\mathrm{AB}$ effects have been found by SotoFaraco and Spence (2002) and Arnell and Larson (2002). Soto-Faraco and Spence embedded T1 and T2 digits in two concurrent streams of letters presented in the visual modality and in the auditory modality. T1 and T2 were presented in either modality unpredictably, and in the same spatial location. In this design, there were equiprobable conditions in which targets were presented in the same modality (either the visual or the auditory modality), or in different modalities (varying unpredictably the modality order). Participants in this experiment were instructed to report T1 and T2 with no speed pressure, and independently of order or modality of presentation, typing them on the keyboard of the computer used for stimuli presentation. Interestingly, whereas no $A B$ effects were found in the 
crossmodal conditions, sizeable $\mathrm{AB}$ effects were found both in the unimodal visual condition and in the unimodal auditory condition. Of relevance in this context, analogous unimodal AB effects have been reported by Arnell and Larson, who asked participants to monitor concurrent visual and auditory streams of stimuli for the presence of one of two prespecified letters (i.e., $\mathrm{K}$ or L). In partial contrast with the results of Soto-Faraco and Spence, however, an $\mathrm{AB}$ effect was found by Arnell and Larson also in the crossmodal condition in which a visual $\mathrm{T} 2$ followed the presentation of an auditory $\mathrm{T} 1$.

Contrary to the many studies mentioned above that focused on $\mathrm{AB}$ effects in vision and audition, pure $\mathrm{AB}$ effects in taction have been the object of much less investigation, with only one published paper focusing specifically on this issue. Hillstrom, Shapiro, and Spence (2002) presented participants with streams of briefly presented (i.e., $150 \mathrm{~ms}$, on average across the experiments) cutaneous vibrations applied to the fingertips. Two target vibrations, $\mathrm{T} 1$ and $\mathrm{T} 2$, were embedded in each tactile stream. In different trials, T1 and T2 were separated by SOAs ranging from about $100 \mathrm{~ms}$ to more than $1 \mathrm{~s}$. In different experiments, T1 and T2 could be selected on the basis of either intensity, duration, frequency, or location, or by a combination of these tactile features. Three of the seven experiments carried out by Hillstrom et al. tested AB effects in the tactile modality, in the absence of various forms of task or feature switches. In these experiments the selection of T1 and T2 occurred on the basis of the same physical feature (intensity, duration, or location, in Exps. 1, 2, and 7, respectively). Furthermore, the features reported from $\mathrm{T} 1$ and $\mathrm{T} 2$ without speed pressure at the end of each trial were the same (one of two prespecified target frequencies in Exps. 1 and 2; one of two prespecified fingers of a given hand in Exp. 7). In all these conditions, participants reported the prespecified feature from both $\mathrm{T} 1$ and $\mathrm{T} 2$ on half of the trials, and only from T2 on the other half of the trials. Interestingly, a clear tactile $\mathrm{AB}$ effect was found only in Experiment 7, where $\mathrm{T} 1$ and $\mathrm{T} 2$ were presented separately to the left and right hands in the form of vibrations to either the index finger or thumb. The task was to report which of these two sensory surfaces had been stimulated. In Experiments 16 report accuracy for T2 decreased, as SOA was reduced, as much when T1 had to be reported as when T1 could be ignored, and almost as much (although statistically less so) in Experiment 7. In other words, unlike what is typically found in vision, there were strong SOA effects in the ignore-T1 condition.

The source of the SOA effects on ignore-T1 trials could be explained in two not mutually exclusive ways. First, it is possible that T1 masked T2. Second, it is possible that participants could not ignore T1, even when instructed to do so. Given that T1 and T2 often had very similar physical features, it is possible that trying to select $\mathrm{T} 2$ led to contingent capture by $\mathrm{T} 1$ 
(Folk, Remington, \& Johnston, 1992). Hillstrom et al. (2002) argued that SOA effects on $\mathrm{T} 2$ report accuracy in the ignore-T1 condition produced by involuntary encoding of $\mathrm{T} 1$ would constitute an $\mathrm{AB}$ effect. Usually, however, one defines the $\mathrm{AB}$ as the difference in accuracy of report of $\mathrm{T} 2$ between the control condition (ignore T1) and the experimental condition (encode T1), which provides unambiguous evidence for an $\mathrm{AB}$ when there is a significant difference, at short SOA, between the two experimental and control conditions, and a smaller difference or no difference at long SOA. In the present paper we sought to explore the issue of this reduced $A B$ effect in taction by varying orthogonally two different dimensions related to the sequential tactile stimulation.

In the present designs, $\mathrm{T} 2$ consisted of the stimulation of two fingers (index and middle) on either the right or the left hand. The stimulation sequence consisted of a pulse to one finger, followed by a pulse to the other finger, followed by simultaneous pulses to both fingers. With equal probability, the stimulation sequence started with the index or middle finger. A second stimulus sequence of the same type was applied to the other hand after an SOA of either $360 \mathrm{~ms}$ or $800 \mathrm{~ms}$. For report-T1 trial blocks, the task was to report which finger was stimulated first, for each sequence. For ignore-T1 trial blocks, the task was to report which finger was stimulated first in the second sequence (T2). In all four experiments in this paper, we found a reliable $\mathrm{AB}$ defined in the traditional way as a larger effect of SOA for the report-T1 condition than for the ignore-T1 condition.

In addition, across the experiments, we manipulated the similarity of $\mathrm{T} 1$ and $\mathrm{T} 2$ on ignore-T1 trials by varying orthogonally two specific dimensions related to their presentation, namely, whether the order of hand stimulation was unpredictable (left-right and right-left trials intermixed at random) versus predictable (blocked), and the similarity of the patterns defining T1 and T2 (identical vs. different). Based on the results of these manipulations we were able to rule out masking factors as the possible cause of the strong lag effects that were found in the ignore-T1 condition of Hillstrom's et al. (2002) designs, and we discovered that the pattern similarity and the predictability of the order of presentation interacted to allow participants to ignore $\mathrm{T} 1$ when instructed to do so (something they could not do when each cue was presented in isolation).

\section{EXPERIMENT 1}

\section{Method}

Participants. A total of 64 students at the University of Padova volunteered to participate in the following experiments, 16 students in each experiment. All were undergraduate or graduate students, with an age 
ranging from 20 to 32 years. All were naive to the purpose of the experiment, and all reported no deficits in taction.

Stimuli and apparatus. The tactile stimuli were two different triplets of presses applied to the distal pads of the index and the middle fingers. One triplet of presses stimulated the left hand and the other stimulated the right hand. Each press lasted $20 \mathrm{~ms}$. A triplet began with a first press applied to one fingerpad (e.g., of the middle finger), followed by a blank interval of a variable temporal duration (see below). After the blank interval, a second press was applied to the other fingerpad (e.g., of the index finger), followed by a blank interval of the same temporal duration as that of the preceding blank interval. The sequence terminated with a third press applied simultaneously to both fingers. There were two possible patterns of stimulation that differed in terms of which finger was stimulated first (index or middle finger).

The apparatus for the generation of the tactile stimuli consisted of two tactile stimulators, placed on a horizontal surface lying in front of the participant, fitted against the distal pads of the index and middle fingers of each hand. The stimulators were placed symmetrically with respect to the participants' sagittal axis, at a distance of $40 \mathrm{~cm}$ of each other. The stimulators were embedded in foam material to reduce the noise generated by their functioning. Each stimulator consisted of a pair of miniaturised solenoids $(3 \mathrm{~W}, 112 \mathrm{~V})$ with a moving cylindrical metallic plunger $1.4 \mathrm{~mm}$ in diameter and $50 \mathrm{~mm}$ in length. The plunger was oriented perpendicularly to the surface of the skin. The plunger for each stimulator could be activated independently, allowing us to stimulate the index or middle finger of each hand separately. Upon activation, the plunger of a given stimulator moved $2.5 \mathrm{~mm}$ and pressed against the skin of the stimulated finger. A $686 \mathrm{CPU}$ controlled the tactile stimulators, the duration and sequencing of the tactile stimuli, as well as a visual monitor that was used to give instructions and feedback to the participants.

Design and procedure. Participants were seated with both arms resting on the table in front of them, facing a computer monitor. On each trial, two tactile stimuli, T1 and T2, were presented in succession, with each stimulus requiring a distinct response. Each trial began with a pair of horizontally arrayed plus signs $(++)$ displayed at the centre of the monitor, that remained on the screen during the entire trial. The experiment was selfpaced. Participants initiated each trial by uttering the word "go" into a microphone connected to the CPU. After the utterance, a fixed temporal interval of $600 \mathrm{~ms}$ elapsed before the presentation of the first triplet (T1) to the fingers of one hand. At one of two possible SOAs (either 360 or $800 \mathrm{~ms}$ ) following T1, a second triplet (T2) was presented to the fingers of the other 
hand. On each trial, the probability that a given finger was stimulated first was .25; that is, which hand and which finger of a given hand were stimulated first was random and equiprobable. Responses had to be emitted at the end of the stimuli presentation using the " $\mathrm{Z}$ " and " $\mathrm{X}$ " keys of the computer keyboard to indicate which finger of the left hand was stimulated first, and the " $\mathrm{N}$ " and " $\mathrm{M}$ " keys of the computer keyboard to indicate which finger of the right hand was stimulated first. Participants were invited to press the appropriate keys using the same fingers as those subject to the tactile stimulation, maintaining a spatially compatible stimulus-response mapping for the entire duration of the experiment (LEFT HAND: " $\mathrm{Z}$ " = middle; "X" = index; RIGHT HAND: "M" = middle; " $N "=$ index). The computer keyboard was placed on the same surface where the tactile stimulators were positioned, with the sets of adjacent " $Z \& X$ " keys and "N\&M" keys arranged symmetrically with respect to the participant's sagittal axis.

In half of the blocks of trials, participants were instructed to ignore $\mathrm{T} 1$ and to respond only to T2 (i.e., by indicating which finger was stimulated first in the T2 triplet). In the other half of the blocks of trials, participants had to respond to both $\mathrm{T} 1$ and $\mathrm{T} 2$ (i.e., by indicating which finger was stimulated first both in the T1 triplet and in the T2 triplet). When two responses were to be emitted at the end of the trial, no instructions were provided to participants concerning response order. The plus signs displayed at the centre of the screen at the beginning of each trial provided feedback on performance in the previous trial, and acted as a fixation point in the current trial. The left plus sign indicated the performance with the triplet presented to the left hand; the right plus indicated the performance with the triplet presented to the right hand. A plus sign indicated a correct response; a minus sign indicated an incorrect response.

The first portion of the experiment was dedicated to practice. Participants performed four blocks of 8 trials in each T1 condition (i.e., ignore-T1 vs. report-T1). In the first four blocks of practice trials, the SOA between T1 and $\mathrm{T} 2$ was always long. In the second four blocks, T2 was presented at both the long and short SOAs. During the practice phase, the blank temporal duration separating the presses in the T2 triplet was adjusted to keep T2 localisation accuracy off ceiling and above floor, while the blank temporal duration separating the presses in the T1 triplet was maintained constant at $80 \mathrm{~ms}$. Mean accuracy in the task on T2 was computed at the end of each block only for trials at the long SOA. The blank duration for T2 (set initially to $100 \mathrm{~ms}$ ) was lengthened by $10 \mathrm{~ms}$ if accuracy was below $60 \%$, or shortened by $20 \mathrm{~ms}$ if accuracy was above $85 \%$. The staircase procedure continued throughout the entire experiment, using the mean accuracy level on T2 at the long SOA in the previous block of trials in a given T1 condition (that is, there were two concurrent, independent, interleaved staircases, one for 
ignore-T1 trials and one for report-T1 trials), to adjust the T2 blank interval duration for the next block of trials in the same T1 condition. Within each block of trials, the blank interval duration for T2 was held constant. At the end of the practice session, the instructions were repeated, and each participant performed experimental trials organised into eight blocks of 32 trials each. Half of the participants started with four blocks of ignore-T1 trials, followed by four blocks of report-T1 trials. For the other half of the participants, this order was reversed. Levels of SOA, T1 starting location, and $\mathrm{T} 2$ starting location were fully crossed within each block of trials.

\section{Results}

The analyses concentrated on the proportion of correct responses to T1 starting location (in the report-T1 condition), on the proportion of correct responses to T2 starting location, and on $d^{\prime}$ (Green \& Swets, 1974) in the localisation task on $\mathrm{T} 2$ calculated by treating one stimulus category (i.e., index finger) as signal, and the other stimulus category (i.e., middle finger) as noise. Mean proportion of correct responses and $d^{\prime}$ values were analysed using the analysis of variance (ANOVA), in which SOA and T1-task (in the analyses of T2 responses) were treated as a withinsubject variables. A further analysis was carried out on the temporal duration of the blank interval elapsing between the single presses composing the T2 triplet. These durations, averaged over blocks for each participant, were submitted to an ANOVA in which T1-task was treated as a withinsubject variable.

Responses to T1. The mean proportion of correct responses to $\mathrm{T} 1$ at the short SOA and long SOA was .76 and .75, respectively. These values did not differ significantly $(F<1)$.

Responses to T2. The mean proportion of correct responses to T2, as a function of SOA, and as a function of the task performed on T1 is shown in Figure 1 (top-left panel).

The ANOVA performed on the mean proportion of correct responses to T2 revealed a significant effect of SOA, $F(1,15)=159.9, p<.001$, a significant effect of the T1-task, $F(1,15)=18.9, p<.001$, and a significant interaction between these two variables, $F(1,15)=10.2, p<.007$. The SOA effect was significant when the results from the ignore-T1 condition were analysed separately, $F(1,15)=80.5, p<.001$. Analogous results were obtained in the analysis carried out on $d^{\prime}$. The ANOVA revealed a significant effect of SOA, $F(1,15)=260.0, p<.001$, a significant effect of 


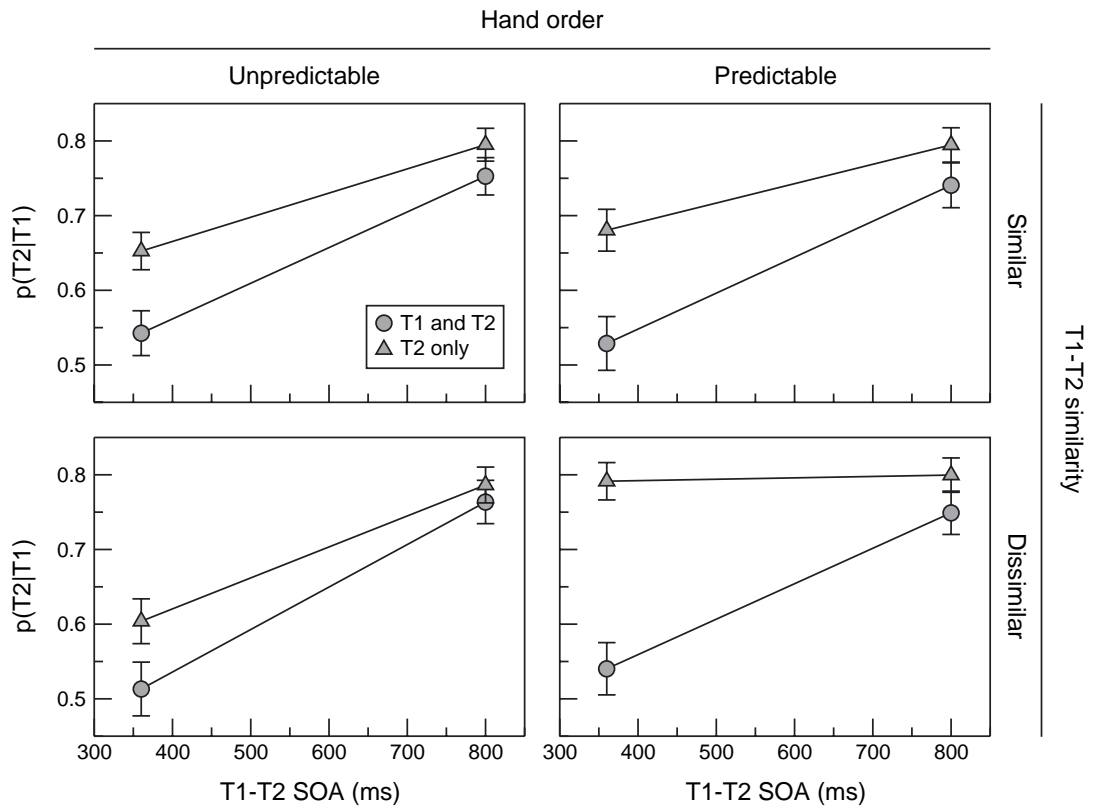

Figure 1. Top-left panel: Results from Experiment 1 (unpredictable hand order, T1 same as T2). Mean proportion of correct responses to T2, as a function of SOA for ignore T1 vs. report-T1 trial blocks. Vertical bars show the standard error of the mean. Top-right panel: Results from Experiment 2 (predictable hand order, T1 same as T2). Bottom-left panel: Results from Experiment 3 (unpredictable hand order, T1 and T2 dissimilar). Bottom-right panel: Results from Experiment 4 (predictable hand order, T1 and T2 dissimilar).

the T1 task, $F(1,15)=11.5, p<.001$, and a significant interaction between these two variables, $F(1,15)=10.7, p<.006$. The SOA effect was significant when the results from the ignore-T1 condition were analysed separately, $F(1$, $1)=115.0, p<.001$.

Rate of stimulation. The mean blank interval durations were $68 \mathrm{~ms}$ in the ignore-T1 and $80 \mathrm{~ms}$ in the report-T1 condition. These values differed significantly, $F(1,15)=5.5, p<.05$. A slightly longer interval was required to bring performance in the desired range when $\mathrm{T} 1 \mathrm{had}$ to be reported. Interestingly, although characterised by easier stimulation conditions (a longer interval between pulses would make it easier to determine which one came first), report-T1 trials nonetheless produced a worse overall performance than ignore-T1 trials. This is reassuring in light of the possible risk that the observed pattern of results could have been actively modulated by the independent manipulation of the staircasing algorithm across ignore-T1 and report-T1 conditions used in Experiment 1. 


\section{Discussion}

The results were clear-cut: The ability to report which finger was stimulated first on the second hand to be stimulated declined as the SOA between hands was reduced, and this decrease was larger when a report had to be made for both hands (T1 and T2) than the first stimulated hand could be ignored (report T2 only, ignore T1). This overall pattern of results demonstrates a purely tactile $\mathrm{AB}$ effect in the absence of task switching, because the same task and selection criteria were used for both targets.

\section{EXPERIMENT 2}

One aspect of the results of Experiment 1 that was expected based on the results reported by Hillstrom et al. (2002) was that, when T1 could be ignored, performance on $\mathrm{T} 2$ was nonetheless clearly reduced as SOA was shortened. In the visual domain, one typically finds relatively small effects of SOA on accuracy of report of T2 when T1 can be ignored. We hypothesised that the unpredictability of the hand of first stimulation might have made it more difficult for participants to ignore T1 and concentrate only on T2. For this reason, in Experiment 2 the hand that was stimulated first was fixed for the entire duration of the test session (e.g., left first, right second), and which hand was stimulated first was counterbalanced across participants. Thus, participants could now predict which hand would be stimulated first and which would be stimulated second. We expected that this might help them to focus on $\mathrm{T} 2$ in blocks of trials where they could ignore T1. This in turn should reduce the magnitude of the SOA effect for $\mathrm{T} 2$ in the ignore-T1 condition.

\section{Method}

Stimuli and apparatus. The same tactile stimuli as those used in Experiment 1 were used in Experiment 2.

Design and procedure. The design and procedure were the same as in Experiment 1 except that which hand was stimulated first was the same on all trials for any given participant. For half of the participants, the order of stimulation was left-right; for the other half the order was right-left.

\section{Results}

Responses to T1. The mean proportion of correct responses to $\mathrm{T} 1$ at the short SOA and long SOA was .73 and .75 , respectively. The small difference between these values was significant, $F(1,15)=7.2, p<.05$. 
Responses to T2. The mean proportion of correct responses to T2, as a function of SOA, and as a function of the task performed on T1 is reported in Figure 1 (top-right panel). An ANOVA performed on the mean proportion of correct responses to T2 revealed a significant effect of SOA, $F(1,15)=72.6, p<.001$, a significant effect of the T1 task, $F(1,15)=41.9$, $p<.001$, and a significant interaction between these two variables, $F(1$, $15)=13.0, p<.003$. The SOA effect was significant when the results from the ignore-T1 condition were analysed separately, $F(1,15)=22.7, p<.001$. The ANOVA carried out on $d^{\prime}$ revealed the same pattern of results: There was a significant effect of SOA, $F(1,15)=61.7, p<.001$, a significant effect of the T1 task, $F(1,15)=29.0, p<.001$, and a significant interaction between these two variables, $F(1,15)=8.7, p<.02$. The SOA effect was also significant when the ignore-T1 condition was analysed separately, $F(1,15)=$ $23.9, p<.001$.

Rate of stimulation. The mean blank interval duration was $90 \mathrm{~ms}$ in the ignore-T1 and $82 \mathrm{~ms}$ in the report-T1 condition. These values differed significantly, $F(1,5)=12.5, p<.005$. These values were reversed compared with what was found in the other three experiments, which suggests that small differences in the mean blank interval do not have large effects on the pattern of results associated with the SOA manipulation, but could explain why the difference between the ignore-T1 and report-T1 conditions is larger in Experiment 2 than in Experiment 1.

\section{Discussion}

As in Experiment 1, the most important results were clear-cut: There was a larger decrease in report accuracy of the second tactile target as SOA was reduced when participants had to process the first tactile target than when they could ignore the first target. Therefore, as in Experiment 1, the results provide evidence for a purely tactile $\mathrm{AB}$ effect affecting stimulus location processing and shows that the results can be replicated under somewhat different experimental conditions, which resemble closely the conditions of Experiment 7 of Hillstrom et al. (2002).

Contrary to our expectations, however, there was little effect of fixing the order of stimulation across the two hands. In Experiment 1 the order of stimulation of the left and right hands was random, whereas the order was constant in Experiment 2. As can be seen in the top two panels of Figure 1, the pattern of results observed in the two experiments was nearly identical. In particular, fixing hand order did not attenuate the SOA effect found in the ignore-T1 condition. It appears that, for our stimulation conditions, it is difficult to ignore a pattern of stimulation on a different hand as the one to 
be reported, even when the participant knows which hand will be stimulated first (and is to be ignored) and which hand will be stimulated second.

\section{EXPERIMENT 3}

The results of Experiments 1 and 2 revealed an interesting inability to filter out unwanted irrelevant tactile stimulation. We found a significant reduction in the probability of correct report of T2 as SOA was reduced, even when T1 could be ignored. Furthermore, this SOA effect was found regardless of whether the order of stimulation of the left and right hands was unpredictable or predictable. With the patterns of stimulation that we used, it appears that it was not possible to ignore completely the first burst of stimulation, which in turn likely caused some interference with the processes mediating the report of $\mathrm{T} 2$.

Spence and McGlone (2001) provided evidence for automatic capture of tactile attention by spatially nonpredictive tactile cues. These authors investigated the ability of participants to localise which of two parts of the hand (either the thumb or the index finger) of either hand received a burst of tactile stimulation. The target tactile stimulus was preceded at SOAs of 200 $\mathrm{ms}, 300 \mathrm{~ms}$, or $400 \mathrm{~ms}$ by a burst of tactile stimulation (i.e., the cue) applied to both fingers concurrently. The cue was applied with equal probability to the hand opposite to the one stimulated by the target, or to the same hand as that stimulated by the target. Interestingly, when the nonpredictive cue was presented to the same hand as that stimulated by the target burst, the response time to localise the stimulated finger was shorter compared to the different condition in which target and cue were presented to different hands. These results from Spence and McGlone suggest that a tactile stimulus may attract attention to the location of the stimulation. Perhaps some, or all, of the SOA effects in the ignore-T1 trials of Experiments 1 and 2 could be due to a reflexive orienting of spatial tactile attention, driven by the representation of the first to-be-ignored stimulus.

On the other hand, research in the visual domain suggests that attentional control settings can modulate the degree to which particular stimuli control involuntary orienting (Folk et al., 1992). In their view, a distractor stimulus is more likely to capture attention if it shares a feature value with a target stimulus on a dimension that is used to select the target among non targets. They call this effect contingent capture. In addition to possible reflexive tactile orienting, we hypothesised that contingent capture may have also played a role in Experiments 1 and 2 because the patterns of stimulation for $\mathrm{T} 1$ and T2 were identical. Thus, it may have been difficult to avoid processing $\mathrm{T} 1$ because it corresponded in all ways (except spatial location) to the stimulation anticipated for the second task. In order to test this 
possibility, in Experiment 3 we introduced a difference in the pattern of tactile stimulation between $\mathrm{T} 1$ and $\mathrm{T} 2$. This patterning difference was designed to provide an additional cue that could be used to filter out the unwanted $\mathrm{T} 1$ signals in ignore-T1 trials.

In the present experiment, as in Experiment 1, the order of presentation of $\mathrm{T} 1$ and $\mathrm{T} 2$ to the two hands was unpredictable. Thus, the only difference between Experiment 1 and Experiment 3 was the difference in the T1 pattern during ignore-T1 blocks, allowing us to compare the results of the two experiments directly. $\mathrm{T} 1$ in the ignore- $\mathrm{T} 1$ condition was composed of a single press applied simultaneously to the index and middle fingers of the same hand, making it temporally and spatially dissimilar from T2. In the report$\mathrm{T} 1$ condition of Experiment 3, T1 and T2 were still composed of triplets of presses (as in Experiments 1 and 2).

\section{Method}

Stimuli and apparatus. In the report-T1 blocks of trials of Experiment 3, the tactile stimulation was identical to the stimulation used in the previous experiments (i.e., both $\mathrm{T} 1$ and $\mathrm{T} 2$ were sequential triplets of brief presses). Unlike in Experiments 1 and 2, the tactile stimulation used as T1 in the ignore-T1 blocks of trials of the present experiment was composed of a single $20 \mathrm{~ms}$ press applied simultaneously to the middle and index fingers of the same hand.

Design and procedure. The design and procedure were the same as in Experiment 1. In particular, as in Experiment 1, the order in which T1 and T2 were applied to the hands was unpredictable.

\section{Results}

Responses to T1. The mean proportion of correct responses to $\mathrm{T} 1$ at the short SOA and long SOA was .75 and .76, respectively. These values did not differ significantly, $F(1,15)=0.25, p>.6$.

Responses to T2. The mean proportion of correct responses to T2, as a function of SOA, and as a function of the task performed on $\mathrm{T} 1$ is reported in Figure 1 (lower-left panel). An ANOVA performed on the mean proportion of correct responses to T2 revealed a significant effect of SOA, $F(1,15)=240.8, p<.001$, a significant effect of the T1 task, $F(1,15)=22.8$, $p<.001$, and a significant interaction between SOA and T1 task, $F(1,15)=$ $5.3, p<.03$. The SOA effect was also significant when the results from the ignore-T1 condition were analysed separately, $F(1,15)=98.1, p<.001$. The ANOVA carried out on $d^{\prime}$ revealed the same pattern of results: There was a significant effect of SOA, $F(1,15)=143.2, p<.001$, a significant, effect of 
T1 task, $F(1,15)=46.4, p<.003$, and a significant interaction between these two variables, $F(1,15)=8.1, p<.02$. The SOA effect was also significant when the results from the ignore-T1 condition were analysed separately, $F(1$, 15) $=70.5, p<.001$.

Rate of stimulation. The mean blank interval durations in the ignore-T1 and report-T1 conditions were $75 \mathrm{~ms}$ and $81 \mathrm{~ms}$, respectively. These values did not differ significantly, $F(1,15)=2.1, p>.2$.

\section{Discussion}

Despite the difference in the pattern of stimulation that we introduced between $\mathrm{T} 1$ and $\mathrm{T} 2, \mathrm{~T} 1$ was evidently still difficult to ignore. Indeed, the size of the SOA effect for the ignore-T1 condition was about the same in Experiment 3 (.15) as it was in Experiments 1 and 2 (mean of .14 across the two experiments). Thus, our expectation that this difference would help participants to ignore $\mathrm{T} 1$ was not supported by the results.

Nonetheless, the results of Experiment 3 provided a further demonstration of a tactile $\mathrm{AB}$ in a form very similar to that put in evidence in the previous experiments. The ability to report which finger was stimulated first on the second hand to be stimulated declined as the SOA between hands was reduced, and this decrease was larger when a report had to be made for both hands than when the first stimulated hand could be ignored.

\section{EXPERIMENT 4}

In Experiment 3, we again found the participants found it difficult to ignore an irrelevant tactile stimulus, even when that stimulus had a different temporal structure from the one to be reported. However, as in Experiment 1 , the order of stimulation of the hands was unpredictable. Perhaps this uncertainty about hand order made it difficult to use the difference in temporal patterning between $\mathrm{T} 1$ and $\mathrm{T} 2$ to construct an effective filter to exclude T1.

In Experiment 4 we provided two different cues to help participants to distinguish $\mathrm{T} 1$ from $\mathrm{T} 2$, and thereby ignore $\mathrm{T} 1$ when it was desirable to do so (in ignore-T1 trial blocks). Although each cue, by itself, was shown to be ineffective in allowing participants to ignore T1 (in Experiments 2 and 3), it is possible that combining the cues would allow participants to achieve something they could not achieve with either cue in isolation. In Experiment 4 , we used the same difference in temporal patterning between T1 and T2 when $\mathrm{T} 1$ was to be ignored, providing one cue to distinguish $\mathrm{T} 2$ from $\mathrm{T} 1$; 
and we made the order of stimulation of the hands predictable, providing another cue based on spatial differences between $\mathrm{T} 2$ and $\mathrm{T} 1$.

By providing both cues simultaneously we tested the hypothesis that participants would be able to combine selection cues to construct a more effective filter than they could with each cue in isolation. Perhaps a form of early selection filtering could be adopted, based on hand of stimulation, as well as a form of late-selection filtering, based on differences in T1 and T2 temporal patterning.

\section{Method}

Stimuli and apparatus. The same tactile stimuli as those used in Experiment 3 were used in Experiment 4. That is, in the report-T1 blocks of trials of Experiment 4, both $\mathrm{T} 1$ and $\mathrm{T} 2$ were sequential triplets of brief presses. As in Experiment 3, T1 in the ignore-T1 trial blocks was composed of a single $20 \mathrm{~ms}$ press applied simultaneously to the middle and index fingers of the same hand.

Design and procedure. The design and procedure were the same as in Experiment 3 except that which hand was stimulated first was the same on all trials for any given participant. For half of the participants, the order of stimulation was left-right; for the other half the order was right-left.

\section{Results}

Responses to T1. The mean proportion of correct responses to $\mathrm{T} 1$ at the short SOA and long SOA was .80 and .79 , respectively. These values did not differ significantly, $F(1,15)=0.52, p>.4$.

Responses to T2. The mean proportion of correct responses to T2, as a function of SOA, and as a function of the task performed on $\mathrm{T} 1$ is reported in Figure 1 (lower-right panel). An ANOVA performed on the mean proportion of correct responses to T2 revealed a significant effect of SOA, $F(1,15)=148.4, p<.001$, a significant effect of the T1 task, $F(1,15)=$ $149.8, p<.001$, and a significant interaction between these two variables, $F(1,15)=39.1, p<.001$. The SOA effect was not significant when the results from the ignore-T1 condition were analysed separately $(F<1)$. The ANOVA carried out on $d^{\prime}$ values revealed the same pattern of results: There was a significant effect of SOA, $F(1,15)=55.4, p<.001$, a significant effect of the T1 task, $F(1,15)=101.0, p<.001$, and a significant interaction between these two variables, $F(1,15)=17.9, p<.001$. The SOA effect on $d^{\prime}$ values was not significant when the results from the ignore-T1 condition were analysed separately $(F<1)$. 
Rate of stimulation. The mean blank interval durations in the ignore-T1 and report-T1 conditions were $73 \mathrm{~ms}$ and $85 \mathrm{~ms}$, respectively. This $12 \mathrm{~ms}$ difference resulted in a significant effect of $\mathrm{T} 1$ task on blank duration, $F(1$, $15)=4.5, p<.05$.

\section{Joint analysis of Experiments 1-4}

The most informative results from all the present experiments (i.e., T2 report accuracy and $d^{\prime}$ values) were analysed as a function of hand order of presentation of $\mathrm{T} 1$ and $\mathrm{T} 2$ (unpredictable: Experiments 1 and 3 vs. predictable: Experiments 2 and 4), T1-T2 similarity (similar: Experiments 1 and 2 vs. dissimilar: Experiments 3 and 4), SOA, and the type of task on $\mathrm{T} 1$. In the present ANOVA, hand-order and $\mathrm{T} 1-\mathrm{T} 2$ similarity were considered as between-subject variables, whereas SOA and T1 task were considered as within-subject variables. Only the results from trials with a correct response to $\mathrm{T} 1$ were included in the analysis.

As one would expect, all the effects that were significant in the analyses of the individual experiments were now even more so. The critical test we were looking were those involving the factors involving the different experiments. Most importantly, the four-way interaction (predictability of hand, similarity of T1 stimulation, SOA, and report vs. ignore T1) approached significance, $F(1,60)=3.0, p<.09$. In addition, three-way interactions between hand-order, T1-T2 similarity, and SOA, $F(1,60)=11.1, p<.002$, and between hand-order, SOA, and task on T1, $F(1,60)=8.2, p<.006$, were also significant. Similar results were obtained in the ANOVA performed on mean $d^{\prime}$ values. The four-way interaction between all factors approached significance, $F(1,60)=3.1, p<.08$. The three-way interactions between hand-order, T1-T2 similarity, and SOA, $F(1,60)=12.7, p<.001$, and between hand-order, SOA, and task on T1, $F(1,60)=4.2, p<.04$, were also significant.

In order to obviate to the apparent lack of power to detect the four-way interaction and is suggested by the patterns of results displayed in Figure 1, the results from report-T1 trials and ignore-T1 trials were considered separately in two subanalyses. An ANOVA carried out on the mean percentage correct localisation responses to $\mathrm{T} 2$ in trials in which $\mathrm{T} 1 \mathrm{had}$ to be reported indicated only the main effect of SOA, $F(1,60)=426.3, p<$ $.001, F<1$, for all other main effects or interactions. The only significant factor in the ANOVA carried out on $d^{\prime}$ values for the same type of trials was also SOA, $F(1,60)=298.2, p<.001, F<1$, for all other main effects or interactions.

An ANOVA carried out on the mean percent of correct localisation responses to $\mathrm{T} 2$ in trials in which $\mathrm{T} 1$ could be ignored indicated the main effects of hand-order, $F(1,60)=6.8, p<.02$, and SOA, $F(1,60)=131.0, p<$ 
.001. The two-way interaction between hand-order and SOA, $F(1,60)=$ 26.7, $p<.001$, and the three-way interaction between hand-order, SOA, and T1-T2 similarity, $F(1,60)=14.2, p<.001$, were significant. Analogous results were obtained in the ANOVA carried out on $d^{\prime}$ values estimated on the basis of the same type of trials. There were main effects of hand-order, $F(1,60)=7.3, p<.01$, and SOA, $F(1,60)=99.2, p<.001$. The two-way interaction between hand-order and SOA, $F(1,60)=19.4, p<.001$, and the three-way interaction between hand-order, SOA, and T1-T2 similarity, $F(1$, $60)=16.2, p<.001$, were significant.

\section{Discussion}

In Experiment 4, accuracy of report of T2 decreased sharply as SOA was reduced for report-T1 trials, but in sharp contrast there was no effect of SOA for ignore-T1 trials. This pattern of results shows that it is possible, under appropriate conditions, to ignore a tactile distractor presented just before a tactile target. This finding argues against a purely automatic form of attentional capture in taction. The absence of an SOA effect for ignore-T1 trials was also in sharp contrast with what was found in Experiments 1, 2, and 3. Evidently, combining a predictable order of hand stimulation and a tactile pattern difference between $\mathrm{T} 1$ and $\mathrm{T} 2$ allowed participants to ignore T1 during ignore blocks. Not surprisingly, because the report-T1 conditions were identical in all four experiments, there was no difference in the magnitude of the SOA effect on accuracy of report of T2 for the report-T1 trial blocks across the four experiments. The results for these equivalent conditions displayed a remarkable stability across experiments, boosting our confidence in the differences we found for the conditions that did differ across experiments, that is, in the ignore-T1 conditions.

\section{GENERAL DISCUSSION}

The results from Experiments 1-4 provide clear-cut and unambiguous demonstrations of an $\mathrm{AB}$ effect produced entirely with tactile stimulation. In these experiments the accuracy of report of an attribute of a second tactile target decreased more as the SOA between the first target (T1) and the second target (T2) was reduced when an attribute of T1 also had to be reported relative to the decrease observed when $\mathrm{T} 1$ could be ignored. This $\mathrm{AB}$ effect was obtained under conditions in which the same task and selection criteria were in effect for $\mathrm{T} 1$ and $\mathrm{T} 2$, ensuring that task switching, and/or switching of selection criteria for $\mathrm{T} 1$ and $\mathrm{T} 2$, could not provide an explanation for the greater effects of SOA in the report-T1 condition than in the ignore-T1 condition. 
These results are important because they suggest that the underlying causes of the $\mathrm{AB}$ effect are likely to be general in the sense that they appear to operate in a similar fashion following visual input (e.g., Raymond, Shapiro, \& Arnell, 1992), auditory input (Arnell \& Jolicœur, 1999; SotoFaraco \& Spence, 2002), and tactile input (as demonstrated herein; see also Hillstrom, Shapiro, \& Spence, 2002). In every case, processing a first target for the purpose of the later report of one of its attributes causes a deficit in the ability to report an attribute of a second target presented at a short SOA relative to the first target. This generality across different input modalities suggests that there is either a single general mechanism that is shared by all studied modalities, or that there are similar within-modality mechanisms that are repeated within each studied modality. The fact that there have been multiple demonstrations of crossmodal AB effects (e.g., Arnell \& Jolicœur, 1999; Arnell \& Larson, 2002; Dell'Acqua, Jolicœur et al., 2003; Dell'Acqua, Turatto, \& Jolicœur, 2001; Jolicœur, 1999b; Soto-Faraco et al., 2002) implies that one or more common supramodal mechanism or resource must sometimes be involved in processing inputs from different sensory modalities.

Having ruled out masking of $\mathrm{T} 2$ by $\mathrm{T} 1$ presentation as a possible cause of the deficits evidenced in Experiments 1-4, one may wonder whether such deficits reflect a true $\mathrm{AB}$ deficit (i.e., a T2 consolidation difficulty), or instead a different form of deficit, perhaps induced by having to attend to spatially different locations. In this alternative perspective, it was a general difficulty to move attention from one hand to the other hand that caused the AB-like pattern of results found in the present study. The assumption in this case would be that participants, on encode-T1 trials, took longer to process $\mathrm{T} 1$ compared to the ignore-T1 condition and that resulted in an increased probability to miss $\mathrm{T} 2$ when $\mathrm{T} 1$ and $\mathrm{T} 2$ were presented in close temporal contiguity. This argument is obviously plausible, but it encounters one major objection. Attention can demonstrably be split between opposite fingers of both hands. Craig (1985, Exps. 4-5) compared the ability of participants to integrate and discriminate tactile patterns presented to either two fingers of the same hand (ipsilateral condition), or to opposite fingers of the different hands (bilateral condition). The to-be-processed tactile patterns were displayed at SOAs varying from $0 \mathrm{~ms}$ to $400 \mathrm{~ms}$, and participants were either warned in advance about the location of presentation of the stimuli, or they received no information about it. Interestingly, although a clear reduction in detection and integration accuracy was observed in the ipsilateral condition, an optimal performance was observed at all SOAs in the bilateral condition, even when participants did not know in advance the order of stimuli presentation. These results, in Craig's (1985) view, implied that participants were able to split their attention almost perfectly between the fingerpads of the two hands (see also Craig, 1989). In our view, these 
results have also implications for the interpretation of the present findings, in that they suggest that the $\mathrm{AB}$ observed in Experiments 1-4 was an encoding deficit at all effects, namely, T2 was missed not because the hand through T2 was delivered was momentarily unattended, but because, as proposed for other within-modality architecture of the $\mathrm{AB}$ effect, $\mathrm{T} 2$ location consolidation was momentarily postponed at the shorter SOA.

Despite strong evidence for crossmodal interactions mediated by a supramodal representation of space (Eimer \& van Velzen, 2002), and involuntary shifts of attention sometimes caused by sudden onsets in a different sensory modality (Turatto, Galfano, Bridgeman, \& Umiltà, 2004; see also Turatto, Benso, Galfano, \& Umiltà, 2002), the exact conditions that lead to crossmodal AB effects remain elusive (Arnell \& Larson, 2002; Chun, \& Potter, 2001; Duncan, Martens, \& Ward, 1997; Potter et al., 1998; SotoFaraco et al., 2002). One candidate capacity-demanding process that has been proposed as a likely common underlying cause of the $\mathrm{AB}$, whether it be within each modality, or a shared supramodal mechanism, is the short-term consolidation of information in STM (Chun \& Potter, 1995; Jolicœur \& Dell'Acqua, 1998). The present results provide additional converging evidence for this possibility.

Although certain stimuli sometimes appear to attract attention involuntarily (particularly sudden onsets), there is good evidence that the degree to which stimuli attract attention - even for sudden onsets - is controlled by an interaction between aspects of the stimulus and the current intentions and goals of the observer. Work by Folk and his colleagues demonstrates nicely that attentional capture can be modulated by top-down attentional set (Folk \& Remington, 1998; Folk et al., 1992; Folk, Remington, \& Wright, 1994). The AB paradigm highlights some aspects of attentional control, indeed, depends on this top-down control because the control condition (i.e., the baseline against which results from the experimental condition are compared) often requires that a salient stimulus be ignored. For example, a control condition often used in the $\mathrm{AB}$ paradigm is to present a distinctive target, as T1, such as a white letter presented in an RSVP stream of grey letters, and to require report only of $\mathrm{T} 2$. When $\mathrm{T} 1$ and $\mathrm{T} 2$ are presented at the same physical location, observers are usually able to process T2 apparently without any cost associated with the mere presentation of a salient T1 (e.g., Raymond et al., 1992). The AB effect is found, in contrast, when the observer must report aspects of both $\mathrm{T} 1$ and $\mathrm{T} 2$. The ability to ignore irrelevant salient stimuli in the $\mathrm{AB}$ paradigm has also been demonstrated for crossmodal situations. For example, Jolicœur (1999b), Exp. 1) found no evidence for a deficit in the processing of a visual T2 following the presentation of a tone for which no response was required. Folk, Leber, and Egeth (2002) examined contingent capture in the context of visual targets presented using RSVP. The distractors were presented at a 
different spatial location (above, below, left, or right of the central RSVP stream). Attentional capture was revealed by a loss of accuracy in the report of the target letter. When targets were selected based on search for a specific colour (e.g., report the red letter presented among letters of other colours), peripheral distractors that matched the target colour (e.g., red) captured attention, but peripheral distractors in other colours did not. The results demonstrate that observers in the experiments of Folk et al. were not able to implement an effective conjunctive input filter to constrain input of a specific feature at a single spatial location.

Isolating the precise conditions under which sensory signals capture attention is a complex theoretical challenge. The present findings provide interesting new insights into this problem in the context of tactile stimulation. In Experiments 1, 2, and 3, observers were less accurate in their reports of the T2 tactile stimulus when T2 followed T1 at short SOA, relative to the long SOA condition even when they were instructed to ignore T1. These results provide evidence of involuntary attentional capture by T1. Interestingly, capture was modulated by an interaction between the predictability of the order of stimulation of the hands and the similarity in the patterns of tactile stimulation between $\mathrm{T} 1$ and $\mathrm{T} 2$, providing evidence for the importance of top-down attentional control settings, as found in the visual domain by Folk and his colleagues. Strong evidence for capture was found except when order of stimulation was predictable (fixed within blocks) and when $\mathrm{T} 1$ and $\mathrm{T} 2$ were dissimilar. Each one of these two factors alone (predictability of order of stimulation, similarity of T1 and T2) was insufficient to prevent capture (Experiments 1, 2, and 3). As in the visual domain, it appears that observers have difficulty in implementing a conjunctive input filter to select a particular type of tactile input only from one location. Indeed, there were no significant differences in the magnitude of the SOA effect in the control condition (T2 only) across Experiments 1, 2, and 3. As in vision, however, when the feature defining $\mathrm{T} 2$ for selection was sufficiently different from the features present in $\mathrm{T} 1$, participants were able implement an effective input filter (Experiment 4). It is possible that larger differences in patterns of tactile stimulation could, by themselves, cause a release from capture, even under conditions of location uncertainty, and this would likely be a fruitful avenue for future research. More generally, modulations of attentional capture could be used as a paradigm to map out the functional dimensions of tactile stimulation. 


\section{REFERENCES}

Allport, D. A., Styles, E. A., \& Hsieh, S. (1994). Shifting intentional set: Exploring the dynamic control of tasks. In C. Umiltà \& M. Moscovitch (Eds.), Attention and performance $X V$ : Conscious and nonconscious information processing (pp. 421-452). Hillsdale, NJ: Lawrence Erlbaum Associates, Inc.

Arnell, K. M., \& Jolicœur, P. (1999). The attentional blink across stimulus modalities: Evidence for central processing limitations. Journal of Experimental Psychology: Human Perception and Performance, 25, 630-648.

Arnell, K. M., \& Larson, J. M. (2002). Cross-modality attentional blinks without preparatory taskset switching. Psychonomic Bulletin and Review, 9, 497-506.

Chun, M. M., \& Potter, M. C. (1995). A two-stage model for multiple target detection in rapid serial visual presentation. Journal of Experimental Psychology: Human Perception and Performance, 21, 109-127.

Chun, M. M., \& Potter, M. C. (2001). The attentional blink and task switching within and across modalities. In K. L. Shapiro (Ed.), The limits of attention (pp. 20-35). New York: Oxford University Press.

Craig, J. C. (1985). Attending to two fingers: Two hands are better then one. Perception and Psychophysics, 38, 496-511.

Craig, J. C. (1989). Interference in localizing tactile stimuli. Perception and Psychophysics, 43, $343-$ 355.

Dell'Acqua, R., Jolicœur, P., Pesciarelli, F., Job, R., \& Palomba, D. (2003). Electrophysiological evidence of visual encoding deficits in a crossmodal attentional blink paradigm. Psychophysiology, 40, 629-639.

Dell'Acqua, R., Pascali, A., Jolicœur, P., \& Sessa, P. (2003). Four-dot masking produces the attentional blink. Vision Research, 43, 1907-1913.

Dell'Acqua, R., Turatto, M., \& Jolicœur, P. (2001). Cross-modal attentional deficits in processing tactile stimulation. Perception and Psychophysics, 63, 777-789.

Donchin, E. (1981). Surprise! ... Surprise? Psychophysiology, 18, 493-515.

Donchin, E., \& Coles, M. G. H. (1988). Is the P300 component a manifestation of context updating? Behavioral and Brain Sciences, 11, 357-374.

Duncan, J., Martens, S., \& Ward, R. (1997). Restricted attentional capacity within but not between sensory modalities. Nature, $387,808-810$.

Eimer, M., \& van Velzen, J. (2002). Crossmodal links in spatial attention are mediated by supramodal control processes: Evidence from event-related potentials. Psychophysiology, 39, 437-449.

Folk, C. L., Leber, A. B., \& Egeth, H. E. (2002). Made you blink! Contingent attentional capture produces a spatial blink. Perception and Psychophysics, 64, 741-753.

Folk, C. L., \& Remington, R. (1998). Selectivity in distraction by irrelevant featural singletons: Evidence for two forms of attentional capture. Journal of Experimental Psychology: Human Perception and Performance, 24, 847-858.

Folk, C. L., Remington, R. W., \& Johnston, J. C. (1992). Involuntary covert orienting is contingent on attentional control settings. Journal of Experimental Psychology: Human Perception and Performance, 18, 1030-1044.

Folk, C. L., Remington, R. W., \& Wright, J. H. (1994). The structure of attentional control: Contingent attentional capture by apparent motion, abrupt onset, and color. Journal of Experimental Psychology: Human Perception and Performance, 20, 317-329.

Giesbrecht, B., \& Di Lollo, V. (1998). Beyond the attentional blink: Visual masking by object substitution. Journal of Experimental Psychology: Human Perception and Performance, 24, $1454-1466$. 
Green, D. M., \& Swets, J. A. (1974). Signal detection theory and psychophysics. Huntington, NY: Krieger.

Hillstrom, A. P., Shapiro, K. L., \& Spence, C. (2002). Attentional limitations in processing sequentially presented vibrotactile targets. Perception and Psychophysics, 64, 1068-1082.

Isaak, M. I., Shapiro, K. L., \& Martin, J. (1999). The attentional blink reflects retrieval competition among multiple rapid serial visual presentation items: Tests of an interference model. Journal of Experimental Psychology: Human Perception and Performance, 25, 1774-1792.

Johnson, R. (1986). A triarchic model of P300 amplitude. Psychophysiology, 23, 367-384.

Jolicœur, P. (1999a). Concurrent response selection demands modulate the attentional blink. Journal of Experimental Psychology: Human Perception and Performance, 25, 1097-1113.

Jolicœur, P. (1999b). Restricted attentional capacity between sensory modalities. Psychonomic Bulletin and Review, 6, 87-92.

Jolicœur, P., \& Dell'Acqua, R. (1998). The demonstration of short-term consolidation. Cognitive Psychology, 36, 138-202.

Jolicœur, P., Dell'Acqua, R., \& Crebolder, J. (2000). Multitasking performance deficits: Forging some links between the attentional blink and the psychological refractory period. In S. Monsell \& J. Driver (Eds.), Attention and performance XVIII: Control of cognitive performance (pp. 309-330). Cambridge, MA: MIT Press.

Juola, J. F., Duvuru, P., \& Peterson, M. S. (2000). Priming effects in attentional gating. Memory and Cognition, 28, 224-235.

Kutas, M., \& Hillyard, S. A. (1980). Event-related brain potentials to semantically inappropriate and surprisingly large words. Biological Psychology, 11, 99-116.

Maki, W. S., Frigen, K., \& Paulson, K. (1997). Associative priming by targets and distractors during rapid serial visual presentation: Does word meanings survive the attentional blink? Journal of Experimental Psychology: Human Perception and Performance, 23, 1014-1034.

Mondor, T. A. (1998). A transient perceptual deficit following selection of an auditory target. Psychonomic Bulletin and Review, 5, 305-311.

Potter, M. C. (1976). Short-term conceptual memory for pictures. Journal of Experimental Psychology: Human Learning and Memory, 2, 509-522.

Potter, M. C., Chun, M. M., Banks, B. S., \& Muckenhoupt, M. (1998). Two attentional deficits in serial target search: The attentional blink and an amodal task-switch deficit. Journal of Experimental Psychology: Learning, Memory, and Cognition, 24, 979-992.

Raymond, J. E., Shapiro, K. L., \& Arnell, K. M. (1992). Temporary suppression of visual processing in an RSVP task: An attentional blink? Journal of Experimental Psychology: Human Perception and Performance, 18, 849-860.

Rogers, R. D., \& Monsell, S. (1995). Costs of a predictable switch between simple cognitive tasks. Journal of Experimental Psychology: General, 124, 207-231.

Rolke, B., Heil, M., Streb, J., \& Hennighausen, E. (2001). Missed prime words within the attentional blink evoke an N400 semantic priming effect. Psychophysiology, 38, 165-174.

Shapiro, K., Driver, J., Ward, R., \& Sorensen, R. E. (1997). Priming from the attentional blink: A failure to extract visual tokens but not visual types. Psychological Science, 8, 95-100.

Soto-Faraco, S., \& Spence, C. (2002). Modality-specific auditory and visual temporal processing deficits. Quarterly Journal of Experimental Psychology, 55A, 23-40.

Soto-Faraco, S., Spence, C., Fairbank, K., Kingstone, A., Hillstrom, A. P., \& Shapiro, K. (2002). A crossmodal attentional blink between vision and touch. Psychonomic Bulletin and Review, 9 , $731-738$

Spence, C., \& McGlone, F. P. (2001). Reflexive spatial orienting of tactile attention. Experimental Brain Research, 141, 324-330.

Sperling, G., Budiansky, J., Spivak, J. G., \& Johnston, M. C. (1971). Extremely rapid visual search: The maximum rate of scanning letters for the presence of a numeral. Science, 174, 307-311. 
Turatto, M., Benso, F., Galfano, G., \& Umiltà, C. (2002). Nonspatial attentional shifts between audition and vision. Journal of Experimental Psychology: Human Perception and Performance, $28,628-639$.

Turatto, M., Galfano, G., Bridgeman, B., \& Umiltà, C. (2004). Space-independent modality driven attentional capture in auditory, tactile and visual systems. Experimental Brain Research, 155, 301-310.

Verleger, R. (1988). Event-related potentials and cognition: A critique of the context updating hypothesis and an alternative interpretation of P3. Behavioral and Brain Sciences, 11, 131-156.

Vogel, E. K., Luck, S. J., \& Shapiro, K. L. (1998). Electrophysiological evidence for a postperceptual locus of suppression during the attentional blink. Journal of Experimental Psychology: Human Perception and Performance, 24, 1656-1674. 